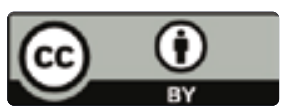

\title{
CARACTERÍSTICAS MATERNAS, FETALES Y NEONATALES DE RIESGO ASOCIADAS A MORTALIDAD NEONATAL
}

\author{
MATERNAL, FETAL AND NEONATAL RISK CHARACTERISTICS ASSOCIATED \\ WITH NEONATAL MORTALITY
}

Huillca-Yalico Cesar Raúl ${ }^{1, a}$, Quispe-Ilanzo Melisa ${ }^{2, b}$

1. Hospital Augusto Hernandez Mendoza - ESSALUD, Ica.

2. Natural and Social Sciences Research

a. Médico Pediatra

b. Licenciada en Enfermería

doi: https://doi.org/10.35563/rmp.v8i2.7

\section{Correspondencia:}

Huillca-Yalico, Cesar Raúl

Dirección: Urb. Las Palmeras

Irg-Ica -Ica

Número de celular: +51 982917808

Correo Electrónico:

dr.huillca@gmail.com

Contribuciones De Autoría:

OTL Contribuciones en la concepción y diseño del manuscrito, recolección, análisis e interpretación de los datos, redacción y revisión crítica del contenido del manuscrito y aprobación final del artículo

Conflicto De Intereses: No declarados.

\section{Financiamiento: Autofinanciado}

\section{Como Citar}

Huillca-Yalico, Cesar Raúl, Quispe-llanzo Melisa. Características maternas, fetales y neonatales de riesgo asociadas a mortalidad neonatal. Rev méd panacea. 2019; (2): 82-86. doi: https://doi.org/10.35563/rmp.v8 i 2.276

Recibido: 11 - 03 - 2019

Aceptado: 11 - 05 - 2019

Publicado: 12 - 05 - 2019

\section{RESUMEN}

Introducción: En los últimos veinte años se han desarrollado esfuerzos por reducir la morbimortalidad perinatal a través de la mejora en los servicios que se ofertan al binomio madre-niño. Objetivo: Determinar las características maternas, fetales y neonatales de riesgo asociadas a mortalidad neonatal Materiales y métodos: Búsqueda bibliográfica, a través de los motores de búsqueda de Pubmed, Sciencedirect, Redalyc, Cochrane, Researchgate, Tripdatabase, Google Scholar y Elsevier, de artículos publicados con una antigüedad no mayor de 5 años, en idioma español como inglés. Discusión: Durante las últimas dos décadas existen esfuerzos por reducir la mortalidad y morbilidad neonatal a través de la mejora en la atención médica, identificando y reduciendo los factores de riesgo. Ante ello, la comunidad científica ha diseñado diversos estudios para determinar los factores maternos y neonatales asociados a la morbi-mortalidad neonatal. Conclusiones: La morbi-mortalidad neonatal es multifactorial y dependerá, en gran manera, de las características de las poblaciones estudiadas.

Palabras clave: mortalidad; morbilidad; recién nacido, factor de riesgo (fuente: DeCS BIREME)

\begin{abstract}
Introduction: In the last twenty years, efforts have been made to reduce perinatal morbidity and mortality through the improvement of the services offered to the mother-child binomial. Objective: To determine the maternal, fetal and neonatal risk characteristics associated with neonatal mortality Methodology: Bibliographic search of published articles, through the search engines of Pubmed, Sciencedirect, Redalyc, Cochrane, Researchgate, Tripdatabase, Google Scholar, and Elsevier, with no more than 5 years old, in Spanish and English. Discussion: During the last two decades, there has been efforts to reduce neonatal mortality and morbidity through improvement in medical care, identifying and reducing risk factors. Given this, the scientific community has designed several studies to determine the maternal and neonatal factors associated with neonatal morbidity and mortality. Conclusions: Neonatal morbidity and mortality is multifactorial and will depend, to a large extent, on the characteristics of the studied populations.
\end{abstract}

Keywords: mortality; morbidity; infant newborn; risk factor (source: MeSH NLM) 


\section{INTRODUCCIÓN}

En los últimos veinte años se han desarrollado esfuerzos por reducir la morbimortalidad perinatal a través de la mejora en los servicios que se ofertan al binomio madre-niño. Estas acciones han tenido un importante impacto en la supervivencia neonatal (1), pero en la actualidad esta mejora muestra un lento avance $(2,3)$. Esta situación cobra vital importancia para la comunidad científica (4), debido a que más de la mitad de los niños que mueren en el primer año de vida son neonatos y más de la mitad, de estas muertes, ocurren en la primera semana de vida $(5,6,7,8)$, identificándose a la prematurez como el principal factor relacionado con esta problemática (9).

La población más vulnerable con limitado acceso a la atención de salud es la depositaria del mayor impacto de este problema (10), lo que indicaría la presencia de otros factores que afectan el desarrollo del producto, durante la gestación y el parto, así como su viabilidad después del nacimiento (11).

En ese sentido, la identificación de factores de riesgo previos al nacimiento así como las intervenciones sanitarias pos-parto ejercen un papel importante en la morbilidad y mortalidad neonatal que debe ser estudiada. Por esta razón, se desarrolla la presente revisión sistemática sobre las características maternas, fetales y neonatales que aumentan el riesgo de mortalidad neonatal en recién nacidos.

\section{MATERIALES Y MÉTODOS}

Se encontraron 11 artículos, dos de ellos realizados en el Perú:

Pérez-Díaz y col.(2018) (12), en su documento "Estudio descriptivo de la mortalidad neonatal en un Hospital Institucional", determinaron las características de la mortalidad neonatal en un Hospital Institucional de México se identificaron 308 defunciones neonatales. La edad gestacional al nacimiento fue de $30 \pm 5$ semanas; el peso al nacer $1944 \pm 990$ gramos. La causa principal de mortalidad fue inmadurez extrema $(58 ; 19 \%)$ seguida de asfixia neonatal $(41 ; 13 \%)$.

Manríquez y Escudero (2017) (13), en su investigación "Análisis de los factores de riesgo de muerte neonatal en Chile 2010-2014", estudiaron los factores sociodemográficos y fisiopatológicos maternos y del recién nacido asociados a la mortalidad neonatal en un hospital terciario de Chile, realizaron un pareamiento en una proporción 1:2 por año, mes de nacimiento y sexo. Durante el período de estudio ocurrieron 81 muertes neonatales, con una tasa estimada de 5,8 por mil nacidos vivos. Se accedió a 65 casos que se compararon con 130 controles. Las principales causas de muerte correspondieron a la prematurez y malformaciones congénitas. Se encontró que la presencia de parto prematuro, recién nacido pequeño para la edad gestacional, puntaje Apgar al minuto entre 4-7, actividad materna fuera del hogar y parto por cesárea fueron los factores de riesgo más prevalentes.
Mendoza y col. (2017) (14), en su estudio "Determinantes biológicos de mortalidad neonatal, en una población de mujeres adolescentes y adultas de un hospital en Colombia" establecieron los determinantes asociados a mortalidad neonatal en una unidad de cuidados intensivos de Colombia. Se incluyó 5567 neonatos vivos, de los cuales fallecieron 125. La mortalidad se presentó en $2,25 \%$ de los neonatos, ocurriendo $76,80 \%$ de las muertes entre 0-7 días. Los determinantes maternos y obstétricos asociados de manera independiente a mortalidad neonatal incluyeron número de visitas en el control prenatal menor a 6 , trabajo de parto prematuro espontáneo, sangrados del tercer trimestre e hipertensión arterial crónica. Entre los determinantes neonatales, la necesidad de reanimación cardiorrespiratoria, prematuridad o bajo peso al nacer, infecciones bacterianas graves, enfermedad de membrana hialina, hipertensión pulmonar, malformaciones congénitas y anomalías cromosómicas y cardiomiopatía hipertrófica, se asociaron independientemente a mortalidad neonatal. Para las complicaciones y eventos adversos hubo asociación independiente y estadísticamente significativa para infecciones asociadas a la atención en salud, hemorragia intraventricular grados III y IV de Papille y hemorragia pulmonar.

Grandia y col.(2016) (15), en el estudio retrospectivo de una cohorte de RNMBP de 26 centros perinatales terciarios y universitarios de la Red Neonatal Sudamericana (NEOCOSUR), que incluye Argentina, Brasil, Chile, Paraguay, Perú y Uruguay, entre 2000 y 2011, denominado "Factores perinatales asociados a la mortalidad neonatal en recién nacidos de muy bajo peso: estudio multicéntrico", evaluó los factores de riesgo asociados a la mortalidad neonatal en recién nacidos de muy bajo peso (RNMBP, $\leq 1500 \mathrm{~g}$ ) y el impacto de la administración antenatal de corticoides. Observaron que -en 11455 registros evaluados- la tasa de mortalidad neonatal fue de $22,3 \%$ con una elevada variabilidad entre los centros. Los factores independientemente asociados a menor mortalidad de los RNMBP fueron la administración de corticoides prenatal, mejor puntaje $Z$ del peso de nacimiento, hipertensión arterial y cesárea. Asimismo, mediante pareamiento, el riesgo de muerte se redujo en $38 \%$ asociado a los corticoides prenatales.

Claros y Mendoza (2016) (16), en su investigación "Impacto de los trastornos hipertensivos, la diabetes y la obesidad materna sobre el peso, la edad gestacional al nacer y la mortalidad neonatal", evaluaron el impacto sobre la mortalidad neonatal, peso al nacer y edad gestacional, de los trastornos hipertensivos (preeclampsia e hipertensión arterial crónica), diabetes (gestacional y no gestacional) y obesidad en la gestación, en un grupo de neonatos nacidos en un hospital colombiano, entre 2005 y 2015. Hallaron asociación significativa entre bajo peso al nacer y prematuridad con preeclampsia e hipertensión arterial 
crónica y entre mortalidad neonatal hipertensión arterial crónica y diabetes.

Carrera y col. (2016) (17), desarrollaron el estudio "Mortalidad neonatal en una institución de tercer nivel de atención" cuantificaron la tasa de mortalidad neonatal en el Instituto Nacional de Perinatología de México, así como las principales causas de muerte y los factores maternos. Incluyó a todos los recién nacidos que fallecieron dentro de los primeros 28 días de vida en el periodo comprendido de enero de 2014 a agosto del 2015 en el Instituto Nacional de Perinatología. La tasa de mortalidad fue del 18,5/1000 NV; la principal causa de muerte fueron las cardiopatías congénitas y problemas respiratorios. El 51,6\% de los fallecimientos ocurrió en las primeras 24 horas de vida, debido a malformaciones genéticas; el 25,4\%, entre el primer y séptimo día, donde predominaron las cardiopatías congénitas y respiratorias y el 22,8\% falleció después de los 7 días de vida. La edad gestacional promedio fue de 31,1 semanas $(23,4-39,1$ semanas) y peso al nacimiento de 1,345 g (390-3,325 g). Mendoza y col. (2015) (18), en el estudio de casos y controles "Influencia de la adolescencia y su entorno en la adherencia al control prenatal e impacto sobre la prematuridad, bajo peso al nacer y mortalidad neonatal" se identificaron la influencia de la adolescencia y su entorno sobre la adherencia al control prenatal y su impacto sobre la prematuridad y/o bajo peso al nacer y la mortalidad neonatal, evaluaron 570 adolescentes y 2093 gestantes $\geq 20$ años de edad, cuyos neonatos fueron hospitalizados en una unidad neonatal. La edad materna 13-19 años se halló asociada a mala adherencia al control prenatal, después de ajustarse por escolaridad $<11$ años, carencia de esposo o compañero permanente, carencia de seguridad social en salud y antecedente de gravidez $\geq 2$ gestaciones. La no adherencia al control prenatal se asoció con aumento de riesgo de prematuridad y/o bajo peso al nacer y de la mortalidad neonatal.

Rangel (2015) (19), en su estudio observacional "Análisis de la mortalidad neonatal en el Hospital General de Ecatepec Dr. José María Rodríguez. Comparación de dos periodos bianuales" identificaron los factores asociados a la mortalidad del recién nacido en el Hospital General de Ecatepec analizaron 188 cuestionarios confidenciales de muerte perinatal de enero 2003 a diciembre 2006 y 100 cuestionarios del período enero 2007 a mayo 2008. La combinación de prematurez y peso menor de 1500 gramos se observó en el $61 \%$ y $66 \%$ de los fallecidos en el primer y segundo período, respectivamente.

Grünebaum y col. (2014) (20), en su trabajo de investigación "Early and total neonatal mortality in relation to birth setting in the United States, 2006-2009" examinaron la mortalidad neonatal en relación con los establecimientos de nacimiento y personal asistente del parto en los Estados Unidos desde 2006 hasta 2009. Se usaron los datos vinculados al nacimiento y la muerte infantil de los Centros para el Control y Prevención de
Enfermedades de los Estados Unidos -desde el 2006 hasta el 2009- para evaluar la mortalidad neonatal total y temprana de productos únicos, eutócicos y a término sin malformaciones congénitas atendidos por parteras y médicos en el hospital así como parteras y otros fuera del hospital. Los investigadores observaron que los partos en casa de parteras tuvieron un riesgo de mortalidad neonatal total significativamente más alto que los partos hospitalarios (1,26 por 1000 vs 0,32 por 1000 nacimientos). Los partos de 41 semanas o más atendidos por parteras (1,84 por 1000 vs 0,27 por 1000) y los partos de primíparas en la casa de las parteras $(2,19$ por 1000 vs 0,33 por 1000) tuvieron riesgos significativamente más altos de mortalidad neonatal total que los partos hospitalarios. En los partos domiciliarios de la partera, la mortalidad neonatal para los primeros partos fue el doble que la de los partos posteriores (2,19 frente a 0,96 por 1000). El exceso de mortalidad neonatal total para los partos domiciliarios por partera en comparación con los partos hospitalarios por partera fue de 9,32 por cada 10000 nacimientos, y el exceso de mortalidad neonatal temprana fue de 7,89 por cada 10000 nacimientos. Los autores concluyeron que el estudio muestra un aumento significativo de la mortalidad neonatal total y temprana en los partos en el hogar e incluso mayores riesgos para las mujeres de 41 semanas o más y las mujeres que tienen un primer parto. Velásquez y col. (2014) (21), en su estudio observacional "Mortalidad neonatal, análisis de registros de vigilancia e historias clínicas neonatales del año 2011 en Huánuco y Ucayali, Perú" estimaron la tasa de mortalidad neonatal y describir las defunciones neonatales ocurridas en el 2011 en hospitales del Ministerio de Salud de las regiones Huánuco y Ucayali revisaron los registros de 185 defunciones neonatales en municipalidades provinciales, direcciones regionales de salud y cuatro hospitales de referencia. La revisión de historias clínicas en los hospitales permitió describir las causas básicas de las muertes neonatales. Hallaron que en el 2011 se reportaron en Huánuco 158 muertes neonatales, con una tasa de 14,5 muertes por 1000 nacidos vivos; en Ucayali, se reportó 138 muertes neonatales, con una tasa de 12,1 muertes por 1000 nacidos vivos. La mayoría de muertes neonatales hospitalarias ocurrieron en los primeros 7 días de vida (87\%), en neonatos prematuros $(73,9 \%)$ y con bajo peso al nacimiento (67\%). Las causas básicas más frecuentes en las muertes neonatales fueron: infección (31,4\%), malformación congénita (22,2\%) y prematuridad (18,9\%).

Vela y col. (2015) (22), en el estudio de casos y controles "Factores de riesgo materno- perinatales asociados a muerte en recién nacidos prematuros con enfermedad de membrana hialina tratados con surfactante pulmonar exógeno; en el Hospital IV Víctor Lazarte Echegaray; Essalud; Trujillo; 2002-2012" determinaron si los factores materno-perinatales fueron factores de riesgo asociados a muerte en recién nacidos prematuros con enfermedad 
de membrana hialina tratados con surfactante pulmonar exógeno en el hospital "Víctor Lazarte Echegaray", EsSalud, Trujillo. Se revisaron 120 historias clínicas, distribuidas en dos grupos: 40 casos que correspondieron a recién nacidos pre-término con enfermedad de membrana hialina tratados con surfactante pulmonar exógeno fallecidos durante su hospitalización y 80 controles que correspondieron a recién nacidos pre-término con enfermedad de membrana hialina tratados con surfactante pulmonar exógeno vivos al momento del alta hospitalaria. Se encontró una diferencia altamente significativa para preeclampsia, depresión severa al nacer, hemorragia intraventricular y peso al nacer $\leq 1500 \mathrm{~g}$. No hubo diferencia significativa para el resto de factores estudiados.

\section{DISCUSIÓN}

Durante las últimas dos décadas los esfuerzos de la investigación médica perinatal y neonatal se ha orientado a reducir la mortalidad y morbilidad neonatal a través de la mejora en la atención médica obstétrica y perinatal, identificando y reduciendo los factores de riesgo. Llevando estos esfuerzo al aumento sustancial en las tasas de supervivencia neonatal, incluso en recién nacidos extremadamente prematuros (1); sin embargo, esta mejora ha mostrado de forma lenta $y$, además, una alta proporción de sobrevivientes experimenta morbilidades neonatales que pueden estar asociadas con resultados adversos a largo plazo $(2,3)$.

La comunidad científica ha diseñado diversos estudios para determinar los factores maternos y neonatales asociados a la morbi-mortalidad neonatal. De los factores neonatales Perez-Diaz y col. (12) postulan que la mortalidad se asocia con la inmadurez neonatal y asfixia; además, Manríquez y Escudero (13) incluyen al APGAR 4-7 al minuto como un factor de riesgo. Para Mendoza y col. (14) no solo estos factores son relevantes sino también el bajo peso al nacer, sepsis neonatal y la malformación crónica, coincidiendo estos resultado con Carrera y col. (17), Rangel (19), Velásquez y col. (21) y Vela y col. (22).

Del lado de los factores maternos Manríquez y col. (13) consideran que la actividad materna fuera del hogar incrementa el riesgo de muerte; además Mendoza y col. (14)(18) postulan que el sangrado en el tercer trimestre de gestación, hipertensión arterial crónica, número de controles prenatales también son factores de riesgo. Finalmente Claros y Mendoza (16) concluyen que la preclamsia y la diabetes mellitus son problemáticas que aun determinan la muerte en esta población.

\section{CONCLUSIÓN}

La morbi-mortalidad neonatal es multifactorial y dependerá, en gran manera, de las características de las poblaciones estudiadas.

Si bien los estudios mostrados no determinan factores fetales asociados con la morbi-mortalidad neonatal, debemos tener en cuenta que las malformaciones congénitas se inician y desarrollan en el periodo gestacional por lo que pueden ser consideradas como factores fetales de riesgo. 


\section{REFERENCIAS BIBLIOGRÁFICAS}

1. Bader D, Kugelman A, Boyko V, Levitzki $O$, Lerner-Geva L, Riskin $A$, et al. Risk factors and estimation tool for death among extremely premature infants: a national study. Pediatrics 2010; 125(4):696-703.

2. Horbar JD, Carpenter JH, Badger GJ, Kenny MJ, Soll RF, Morrow KA, et al. Mortality and neonatal morbidity among infants 501 to 1500 grams from 2000 to 2009 . Pediatrics 2012; 129(6):1019-26.

3. Shah PS, Sankaran K, Aziz K, Allen AC, Seshia M, Ohlsson $A$, et al. Outcomes of preterm infants b29 weeks gestation over 10-year period in Canada: a cause for concern? J Perinatol 2012;32(2):132-8.

4. UN Inter-agency Group for Child Mortality Estimation (UN-IGME). Levels and Trends in Child Mortality. 2015. Disponible en: http://wwwwhoint/maternal_child_adolescent/docum ents/levels_trends_child_mortality_2015/en/

5. Shah S, Zemichael O, Meng H. Factors associated with mortalityand length of stay in hospitalised neonates in Eritrea Africa: Across-sectional study. BMJ Open. 2012; 2:e000792.

6. Worku B, Kassie A, Mekasha A, Tilahun B, Worku A. Predictorsof early neonatal mortality at a neonatal intensive care unitof a specialized referral teaching hospital in Ethiopia. Ethiop JHealth Dev. 2012; 26:200-207.

7. Jacobs J, Kamitsuka M, Clark R, Kelleher A, Spitzer A. Etiologiesof NICU Deaths. Pediatrics. 2015; 1:e59-65.

8. Mukhopadhyay $K$, louis D, Mahajan R, Kumar P. Predictors ofmortality and major morbidities in extremely low birth weightneonates. Indian Pediatr. 2013; 50:1119-20.

9. Harmon QE, Basso O, Weinberg CR, Wilcox AJ. Two denominators for one numerator: the example of neonatal mortality. European Journal of Epidemiology. 2018; PMID:29516296

10. Wou K, Ouellet MP, Chen MF, Brown RN. Comparison of the aetiology of stillbirth over five decades in a single centre: a retrospective study. BMJ Open. 2014; 4(6):e004635.

11. Aquilar A. Consideraciones sobre la mortalidad infantil y su componente neonatal. Arch Argent Pediatr 2016; 114(5):396-401.

12.Pérez-Díaz $R$, Rosas-Lozano $A L$, Islas-Ruz FG, Baltazar-Merino RN, Mata-Miranda MP. Estudio descriptivo de la mortalidad neonatal en un Hospital Institucional. Acta Pediatr Mex. 2018; 39(1):23-32.

13. Manríquez G, Escudero C. Análisis de los factores de riesgo de muerte neonatal en Chile, 2010-2014. Rev Chil Pediatr. 2017; 88(4):458-464.

14. Mendoza LA, Gómez D, Gómez D, Osorio MA, Villamarín EA, Arias MD. Determinantes biológicos de mortalidad neonatal, en una población de mujeres adolescentes y adultas de un hospital en Colombia. Rev Chil Obstet Ginecol 2017; 82(4):424-437.

15. Grandia C, González A, Zubizarretac J, Red Neonatal NEOCOSUR. Factores perinatales asociados a la mortalidad neonatal en recién nacidos de muy bajo peso: estudio multicéntrico. Arch Argent Pediatr 2016; 114(5):426-433.

16. Claros DI, Mendoza LA. Impacto de los trastornos hipertensivos, la diabetes y la obesidad materna sobre el peso, la edad gestacional al nacer y la mortalidad neonatal. Rev Chil Obstet Ginecol. 2016; 81(6):480-488.

17. Carrera S, Hernández M, Fernández LA, Cordero G, Corral E, Barrera PI, et al. Mortalidad neonatal en una institución de tercer nivel de atención. Perinatol Reprod Hum. 2016;30(3):97-102.

18. Mendoza LA, Arias MD, Peñaranda CB, Mendoza LI, Manzano S, Varela AM. Influencia de la adolescencia y su entorno en la adherencia al control prenatal e impacto sobre la prematuridad, bajo peso al nacer y mortalidad neonatal. Rev Chil Obstet Ginecol. 2015; 80(4):306-315.

19. Rangel MN. Análisis de la mortalidad neonatal en el Hospital General de Ecatepec "Dr. José María Rodríguez". Comparación de dos periodos bianuales. Perinatol Reprod Hum. 2015; 29(3):90-94.

20. Grünebaum A, McCullough LB, Sapra KJ, et al. Early and total neonatal mortality in relation to birth setting in the United States, 2006-2009. Am J Obstet Gynecol 2014; 210:e1-e7.

21. Velásquez JE, Kusunoki $L$, Paredes TG, Hurtado $R$, Rosas AM, Vigo WE. Mortalidad neonatal, análisis de registros de vigilancia e historias clínicas neonatales del año 2011 en Huánuco y Ucayali, Perú. Rev Peru Med Exp Salud Publica. 2014; 31(2):228-36.

22. Vela J, Vela J, Panta O, Sánchez V, Delgado M, Vela J. Factores de riesgo materno- perinatales asociados a muerte en recién nacidos prematuros con enfermedad de membrana hialina tratados con surfactante pulmonar exógeno; en el Hospital IV Víctor Lazarte Echegaray; Essalud; Trujillo; 2002-2012. Revista Médica de Trujillo. 2015; 11(2):e1-e21.

\section{PANACEA}

